\title{
Projective Systems and Higher Weights
}

\author{
Hans Georg Schaathun \\ Department of Informatics \\ University of Bergen \\ Høyteknologisenteret \\ N-5020 Bergen, Norway \\ e-mail: georg@ii.uib.no
}

\begin{abstract}
We use projective multisets (projective systems) to find upper bounds on the weight hierarchies for a special class of codes, namely the extremal non-chain codes. Several code constructions exist meeting the bounds with equality.
\end{abstract}

\section{INTRODUCTION}

Let $C$ be a linear $q$-ary code of dimension $k$ and length $n$. The weight $w(S)$ of a subcode $S \subseteq C$, is the number of positions where at least one word in $S$ differs from zero. The $r$ th generalised Hamming weight $d_{r}$ of $C$ is the least weight of an $r$-dimensional subcode of $C$. The sequence $\left(d_{1}, d_{2}, \ldots, d_{k}\right)$ is called the weight hierarchy of $C[6]$.

\section{Extremal Non-Chain Codes}

The chain condition was introduced in [7], and states that there is a chain $D_{0} \subset \ldots \subset D_{k}$ of subcodes, where $D_{i}$ has dimension $i$ and weight $d_{i}$.

The opposite extreme are the extremal non-chain codes, defined as follows. For each pair $(i, j)$ where $1 \leq i<j<k$, there are no subcodes $D_{i} \subseteq D_{j}$ of dimensions $i$ and $j$ respectively such that $w\left(D_{i}\right)=d_{i}$ and $w\left(D_{j}\right)=d_{j}$. The extremal non-chain codes were introduced by Chen and Kløve [1], and this study continues their work.

\section{Projective Multisets}

Let $G$ be a $k \times n$ generator matrix of $C$. By permuting columns of $G$ or by multiplying certain columns by non-zero scalars, we get an equivalent code. Equivalent codes have the same weight hierarchy.

Let $\mathrm{PG}(k-1, q)$ be the projective $(k-1)$-space over the finite field with $q$ elements. The code $C$ is determined up to equivalence by giving the map $\gamma: \mathrm{PG}(k-1, q) \rightarrow\{0,1, \ldots\}$, saying how many times each projective point occurs as a column in $G$. Such a map is called a projective multiset [2], a projective system [5], or a value assignment $[1,4]$. The definition of $\gamma$ is extended by $\gamma(S)=\sum_{x \in S} \gamma(x)$ for all $S \subseteq \mathrm{PG}(k-1, q)$. The number $\gamma(S)$ is called the value of $S$.

We know $[3,5]$ that a subcode $D_{r}$ of dimension $r$ and weight $w$, corresponds to a subspace $S_{r} \subseteq \mathrm{PG}(k-1, q)$ of dimension $k-r-1$ and value $\gamma\left(S_{r}\right)=n-w$. Hence a subcode $D_{r}$ of minimum value corresponds to a projective subspace $S_{r}$ of maximum value. Also if $D_{r} \subseteq D_{r^{\prime}}$, then $S_{r} \supseteq S_{r^{\prime}}$.

The difference sequence $\left(\delta_{0}, \delta_{1}, \ldots, \delta_{k-1}\right)$ is defined by $\delta_{i}=$ $d_{k-1}-d_{k-1-i}$. The difference sequence is easily computed from the weight hierarchy and vice versa. If $S$ is an $i$-space of maximum value, then $\gamma(S)=\delta_{0}+\delta_{1}+\ldots+\delta_{i}$. A difference sequence corresponding to an extremal non-chain code is called an ENDS (Extremal Nonchain Difference Sequence).

\section{RESULtS}

Theorem 1 (General Bound) If $\left(\delta_{0}, \delta_{1}, \ldots, \delta_{k-1}\right)$ is an ENDS, $1 \leq m \leq k-2$, then

$$
\delta_{m} \leq q^{m} \delta_{0}-\frac{q^{m+1}-1}{q-1} .
$$

If equality holds for $m=\bar{m}$, then equality holds for all $m<\bar{m}$.

Theorem 2 (Binary Codes) If $\left(\delta_{0}, \delta_{1}, \ldots, \delta_{k-1}\right), k \geq 4$ is a binary ENDS, then

$$
\delta_{k-2} \leq 2^{k-3} \delta_{1}-2-2^{k-3} .
$$

Theorem 3 (Total Value) If $\left(\delta_{0}, \delta_{1}, \ldots, \delta_{k-1}\right), k \geq 3$ is an ENDS, then

$$
\gamma(\mathrm{PG}(k-1, q)) \leq \sum_{i=0}^{m-1} \delta_{i}+\left(\delta_{m}-1\right) \frac{q^{k-m}-1}{q-1},
$$

for all $m$ such that $1 \leq m \leq k-2$.

Explicit constructions meeting the bounds with equality exist in dimension 5 and less, provided $\delta_{0}$ is sufficiently large; $\delta_{0} \geq 5$ is sufficient in all cases.

\section{ACKNOWLEDGMENTS}

Most of this work is part of my graduate thesis. I am grateful to prof. Torleiv Kløve for all his help as a superviser in all stages of the work.

\section{REFERENCES}

[1] Wende Chen and Torleiv Kløve. Bounds on the weight hierarchies of extremal non-chain codes of dimension 4. Applicable Algebra in Engineering, Communication and Computing, 8:379-386, 1997.

[2] S. Dodunekov and J. Simonis. Codes and projective multisets. Electron. J. Combin., 5(1), 1998. Research Paper 37.

[3] Tor Helleseth, Torleiv Kløve, and Øyvind Ytrehus. Generalized Hamming weights of linear codes. IEEE Trans. Inform. Theory, 38(3):1133-1140, 1992.

[4] Hans Georg Schaathun. Upper bounds on weight hierarchies of extremal non-chain codes. Technical Report 171, Department of Informatics, University of Bergen, 1999. Also available at http://www.ii.uib.no/ georg/sci/inf/coding/public/.

[5] Michael A. Tsfasman and Serge G. Vladut. Geometric approach to higher weights. IEEE Trans. Inform. Theory, 41(6, part 1):1564-1588, 1995. Special issue on algebraic geometry codes.

[6] Victor $K$. Wei. Generalized Hamming weights for linear codes. IEEE Trans. Inform. Theory, 37(5):1412-1418, 1991.

[7] Victor K. Wei and Kyeongcheol Yang. On the generalized Hamming weights of product codes. IEEE Trans. Inform. Theory, $39(5): 1709-1713,1993$. 